\title{
Étude du lien entre deuil, dépression et troubles somatiques auprès d'une population d'immigrés ouest-africains en Europe
}

Association between Mourning, Depression and Somatic Symptoms. Results from a Sample of West African Immigrants in Europe

Estudio de la relación entre el duelo, la depresión y los trastornos somáticos entre una población de inmigrantes de África Occidental en Europa

Cyrille Kossigan Kokou-Kpolou, Daniel Mbassa Menick, Charlemagne Simplice Moukouta et Élodie Gaëlle Ngameni

\section{(2) OpenEdition}

Édition électronique

URL : https://journals.openedition.org/remi/10930

DOI : 10.4000/remi. 10930

ISSN : $1777-5418$

Éditeur

Université de Poitiers

Édition imprimée

Date de publication : 1 octobre 2018

Pagination : 159-186

ISBN : 979-10-90426-62-7

ISSN : 0765-0752

\section{Référence électronique}

Cyrille Kossigan Kokou-Kpolou, Daniel Mbassa Menick, Charlemagne Simplice Moukouta et Élodie Gaëlle Ngameni, «Étude du lien entre deuil, dépression et troubles somatiques auprès d'une population d'immigrés ouest-africains en Europe », Revue européenne des migrations internationales [En ligne], vol. 34 - n² et 3 | 2018, mis en ligne le 01 janvier 2021, consulté le 07 décembre 2022. URL : http://journals.openedition.org/remi/10930 ; DOI : https://doi.org/10.4000/remi.10930 


\section{Étude du lien entre deuil, dépression et troubles somatiques auprès d'une population d'immigrés ouest-africains en Europe}

\section{Cyrille Kossigan Kokou-Kpolou', Daniel Mbassa Menick ${ }^{2}$, Charlemagne Simplice Moukouta ${ }^{3}$ et Élodie Gaëlle Ngameni ${ }^{4}$}

\section{Introduction}

Le deuil d'un être aimé est l'un des événements de vie difficiles à vivre (Bonnano, 2004; Holmes et Rahe, 1967; Rando, 1993). En général, il s'accompagne de perturbations transitoires au niveau biopsychosocial chez la personne endeuillée. Mais faute de ressources intra et interpersonnelles adéquates, ces perturbations peuvent devenir sévères et chroniques. Plusieurs concepts désignent ce syndrome : deuil compliqué (Prigerson et al., 1995), deuil complexe persistant (DSM-55 ${ }^{5}$ APA, 2013), deuil prolongé (CIM-116, Maciejewski et al., 2016). Le deuil compliqué est caractérisé par des sentiments de choc, de tristesse, de colère persistante, par des difficultés à accepter la perte, par la détérioration de sa vie sociale et professionnelle au-delà d'une période moyenne de six mois. Selon plusieurs études, 10 à $25,4 \%$ de la population générale seraient confrontés au deuil compliqué (Lundorff et al., 2017; Newson et al., 2011). Cette prévalence varie considérablement. Craig et al. (2008) ont trouvé dans une population de réfugiés aux États-Unis que cette prévalence était de $54 \%$. Ce qui indique que dans le contexte migratoire, le deuil revêt davantage une charge potentiellement

\footnotetext{
1 Psychologue clinicien, docteur en psychologie clinique, Université de Picardie Jules Verne, UFR Sciences Humaines et Sociales, Philosophie, 1 chemin du Thil, CS52501, 80025 Amiens cedex 1; kossigan.kokou@u-picardie.fr

2 Psychiatre, psychocriminologue-victimologue, praticien hospitalier au pôle $93 \mathrm{G} 15$ de Psychiatrie Adulte, EPS de Ville-Evrard, 93330 Neuilly-sur-Marne; menickfr@yahoo.fr

3 Psychologue clinicien, maître de conférences en psychopathologie clinique à l'Université de Picardie Jules Verne, UFR Sciences Humaines et Sociales, Philosophie, 1 chemin duThil, CS52501, 80025 Amiens cedex 1; moukouta_cs@ hotmail.com

4 Psychiatre, DFMSA Psychiatrie, Groupe Hospitalier du Havre Hôpital Pierre Janet, 47 rue deTourneville, 76600 Le Havre; ngamenielodie@gmail.com

5 DSM : Diagnostic and Statistical Manuel of Mental Disorders (cinquième édition).

6 CIM : Classification Internationale des Maladies (onzième édition, 2018).
} 
traumatique, associée au trouble de stress post-traumatique et à des plaintes somatiques (Hinton et al., 2013a). Malgré cette incidence du deuil en contexte migratoire, l'examen de la littérature révèle que très peu d'études empiriques ont porté sur la problématique du deuil (Hinton et al., 2013a; Panagiotopoulosa et al., 2013; Rachédi et al., 2010), notamment ses liens plausibles avec la dépression et les troubles somatiques. En France, l'on peut noter la rareté des études portant sur cette question, en l'occurrence auprès des migrants et des réfugiés ouest-africains (Bass, 2014). La présente étude contribue à combler quelque peu ce vide. Elle vise à examiner l'impact du deuil d'un parent selon les caractéristiques sociodémographiques et migratoires et à vérifier le lien entre la sévérité du deuil et les symptômes dépressifs et somatiques auprès d'une population d'immigrés togolais résidant en France et en Belgique.

\section{Contextualisation de l'étude}

Pays francophone ouest-africain, le Togo forme une bande étirée entre le Ghana à l'ouest et le Bénin à I'est. II est bordé au sud par l'Océan Atlantique (le golfe de Guinée) et limité au nord par le Burkina Faso. D'un point de vue historique, le Togo et le Cameroun sont les deux pays de l'Afrique qui ont connu successivement une triple domination coloniale : allemande, anglaise ${ }^{7}$ et française (Gayibor, 2011). L'histoire du Togo avant et après l'indépendance en 1960 est marquée par des tensions et des violences politiques, notamment au cours des périodes électorales (Améganvi, 2011; Sarkin et Tetevi, 2017). Ces tensions et ces violences politiques, accompagnées de leur lot de perte en vies humaines, d'arrestations arbitraires, de torture physique et morale et des persécutions politiques, étaient à l'origine de trois grandes vagues d'exil (Rapport Commission Vérité, Justice et Réconciliation, 2012). La première vague d'exil est consécutive aux violences qui ont suivi la première élection présidentielle en 1958. La deuxième vague est intervenue au début des années 1990, dans le contexte des mouvements de démocratisation marqués entre autres par des élections législatives (1991 à 1999) et présidentielles (1998) contestées. Enfin, la dernière vague d'exil est intervenue à la suite à l'élection présidentielle de 2005, violemment réprimée et marquée par de nombreuses pertes en vies humaines (500 selon les Nations unies, 1000 selon les sources locales; voir Rapport Commission Vérité, Justice et Réconciliation, 2012). Le Togo compte 7 millions d'habitants dont $60 \%$ sont âgés de moins de vingt-cinq ans. II n'y a pas de données statistiques sur le nombre de réfugiés togolais, mais la diaspora togolaise est estimée à environ 1500000 de migrants, soit environ un quart de la population générale. II n'existe pas non plus à notre connaissance des études dans le champ de la psychopathologie ayant porté sur cette catégorie de la population.

L'exil est défini comme une migration involontaire, on parle d'immigration forcée (Berry et Sam, 1997; Bhugra et Gupta, 2011). Qu'il s'agisse d'un réfugié politique ou de guerre, la Convention de Genève du 28 juillet 1951 le définit comme une personne qui fuit une situation d'insécurité vitale dans son pays d'origine ou de résidence habituelle. Cette situation d'insécurité peut être une

7 Après la Grande Guerre, le Togo fut placé sous mandat de la Société des Nations avec deux tutelles: I'une "Togo français" et une autre à l'ouest "British Togo ". Cette administration franco-anglaise fut soldée par le partage du territoire : deux tiers du territoire à la France et un tiers au Royaume-Uni. 
menace d'ordre politique, religieux ou ethnique. Son projet migratoire, s'il en était un, est de trouver un refuge, une terre protectrice qui lui assure une sécurité physique et morale. Sa préoccupation immédiate est de sauver sa vie. Ce qui ne semble pas être le cas d'un migrant, souvent dit économique - même s'il est difficile d'en établir les nuances - qui porte un projet directement lié à une quête d'accomplissement professionnel et personnel (Berry et Sam, 1997). Ce projet est également lié à la possibilité d'un retour imminent au pays d'origine contrairement au réfugié dont la possibilité de retour est incertaine. Selon Grinberg et Grinberg (1989), cette difficulté à concevoir la possibilité du retour chez le réfugié explique à moyen terme son sentiment de nostalgie. Dans tous les cas, la trajectoire migratoire est en général marquée par des pertes (e.g., Craig et al., 2008; Hinton et al., 2013a et 2013b) plus ou moins violentes et traumatisantes.

\section{Deuil migratoire ou traumatisme migratoire et psychopathologie}

En psychopathologie, l'immigration a été étudiée, par analogie, à travers les modèles de deuil (deuil migratoire) et de trauma (traumatisme migratoire). En d'autres termes, l'expérience migratoire partagerait certains mécanismes et manifestations similaires avec le deuil d'un être cher et le traumatisme psychique. Grinberg et Grinberg (1989) ont théorisé cette expérience comme étant marquée de multiples ruptures (géographique, temporelle, culturelle, relationnelle et linguistique). Tout autant que le deuil d'un être cher, l'expérience migratoire est caractérisée par de vifs sentiments de nostalgie, de solitude, de douleur parfois intense, pouvant entraîner des affects dépressifs. En s'appuyant sur l'approche de transition psychosociale du deuil de Parkes, d'autres auteurs (e.g., Casado et al., 2010 ; Casado et Leung, 2001 ; Lee, 2010) ont décrit l'immigration comme une expérience douloureuse marquée par plusieurs pertes (pertes de la famille et de la communauté, perte de la langue, perte des modèles culturels du comportement, perte de contact avec le groupe ethnique, etc.). Casado et Leung (2001) ont proposé le concept du "deuil migratoire/migratory grief" pour décrire cette expérience. En France, Nathan (1986) avait utilisé le concept de "traumatisme migratoire", repris par d'autres auteurs à l'instar de Baubet et Moro (2013). Le traumatisme migratoire désigne l'ensemble des désorganisations psychiques et identitaires associées à l'expérience migratoire. Celles-ci n'entraînent pas toujours des effets pathogènes immédiats (Baubet et Moro, 2013; Nathan, 1986; Tousignant, 1992). En somme, ces différentes études n'ont pas examiné le lien entre le deuil consécutif à la mort d'un être aimé et l'expérience migratoire. Elles présentent néanmoins l'intérêt d'avoir montré que le deuil d'un être cher en contexte migratoire peut réactiver les traces traumatiques liées à l'immigration.

En effet, de nombreux facteurs peuvent rendre l'expérience migratoire potentiellement traumatique. Ces facteurs peuvent être classés en deux catégories : d'une part, les traumas pré-migratoires, en l'occurrence les circonstances de départ, les conditions de voyage, etc. (Li, 2016); d'autre part, les stress post-migratoires, comme les procédures administratives (celles d'asile pour les réfugiés), les stress acculturatifs, les conditions socioéconomiques précaires, les difficultés d'emploi, l'isolement social (Berry et Sam, 1997; Bhugra, 2004; Gerritsen et al., 2006; Laban et al., 2005; Li, 2016; Robjant et al., 2009). Le lien entre ces facteurs migratoires et la santé mentale des migrants a fait l'objet de 
plusieurs études (e.g., Bhugra et Gupta, 2011 ; Carswell et al., 2011 ; Carta et al., 2005; Craig et al., 2008; Levecque et Van Rossem, 2015; Missinne et Bracke, 2012; Pumariega et al., 2005). Par exemple, l'étude de Missinne et Bracke (2012) menée dans vingt-trois pays européens a examiné le lien entre les symptômes dépressifs et certaines catégories de population : les migrants et les minorités ethniques. Au rang des pays ouest européens, les migrants en France et aux Pays-Bas avaient manifesté un niveau élevé de symptômes dépressifs comparé aux "autochtones". Concernant l'ensemble des pays enquêtés, lorsque l'effet cumulé du sexe, de l'âge et de la présence du partenaire de vie conjugale est contrôlé, les migrants de la première génération sont plus vulnérables aux risques des symptômes dépressifs. Les résultats ont aussi montré que les migrants moins scolarisés, les sans-emplois, les migrants ayant des difficultés financières étaient enclins aux symptômes dépressifs. Dans une autre étude récente menée dans vingt pays européens, Levecque et Van Rossem (2015) ont examiné le lien entre l'intégration des immigrés européens et non européens (premières et deuxièmes générations) et la dépression. Cette étude a confirmé les résultats de l'étude de Missinne et Bracke (2012). Cependant, ils n'ont pas observé que les immigrés des pays comme la Belgique, la France, le Pays-Bas étaient plus enclins à la dépression, mais c'étaient plutôt les immigrés des pays de l'Europe de l'Est. Carta et al. (2005) ont, quant à eux, effectué une revue des études menées en Europe, ayant examiné le lien entre les déterminants associés à l'immigration et la santé mentale des migrants. Cet examen de la littérature a permis de montrer qu'outre les facteurs liés à l'émigration, les difficultés d'accès aux services de soins, le deuil d'un parent proche constitue un des facteurs stressants associés à des troubles psychiques chez les migrants.

Dans une étude réalisée auprès des réfugiés cambodgiens aux États-Unis, le deuil d'être aimé a reçu une attention particulière (Hinton et al., 2013a). II s'agit des réfugiés ayant survécu au génocide de Pol Pot et dont I'élaboration des traumatismes et des deuils était difficile. Les participants avaient rapporté plusieurs types de deuils à la fois, vécus lors de ce génocide : deuil d'un frère ou d'une sœur, le deuil d'un parent et le deuil d'un enfant. Les résultats ont montré que $76 \%$ des participants avaient manifesté des souvenirs douloureux au cours du mois précédent l'enquête. Ces souvenirs étaient fortement associés à la détresse émotionnelle et somatique ainsi qu'à la sévérité du trouble de stress post-traumatique. Ces souvenirs douloureux et traumatiques étaient souvent revécus à travers des rêves. Les participants avaient perçu ces morts comme une "mauvaise mort", faute de supports rituels adéquats pour donner un sens à ces morts. Hinton et al. (2013a) ont ainsi proposé de prendre en compte des éléments anthropologiques, notamment les rituels de deuil pour mieux expliquer le processus du deuil d'être cher dans le contexte migratoire. Rappelons que la question sur les bénéfices psychologiques des rituels de deuil a été largement abordée dans la littérature. Les résultats des travaux empiriques qui y ont été consacrés, surtout en 1970 et 1990 restent mitigés (e.g., Bolton et Camp, 1987; Euster, 1991 ; Thomas, 1985).

Comme il nous est donné de le constater, peu d'études ont examiné le lien entre le deuil et la santé mentale en situation migratoire. En France, il existe quelques travaux basés sur des cas cliniques qui ont élucidé les difficultés dans la prise en charge des deuils pathologiques chez les migrants africains (Kokou-Kpolou et al., 2016; Moukouta et al., 2016). Ces difficultés s'expliquent 
en partie par les représentations collectives autour de la mort en Afrique, ainsi que par le manque de supports rituels, car dans les sociétés africaines, comme ailleurs, deuil et rituels sont indissociables. Dans les cas cliniques respectifs présentés et analysés dans ces études, les auteurs ont souligné comment les plaintes somatiques brouillaient le tableau symptomatologique, ce qui $n^{\prime}$ est pas de nature à faciliter un diagnostic précis. Adansikou et al. (2017) ont souligné l'importance de prendre en considération la question de la somatisation lorsque I'on est amené à examiner le phénomène de la dépression auprès des sujets africains. II s'agit là d'un aspect intéressant qui jusqu'alors n'avait pas encore été rigoureusement exploré. À notre connaissance, seule l'étude de Sanz (2012), en Suisse, a tenté d'éclairer le lien entre le deuil et la somatisation chez les migrants. Sanz (2012) a utilisé une méthode qualitative basée sur trois études de cas (deux Bosniaques et un Albanais) pour expliquer ce lien. L'auteur a effectivement observé des plaintes somatiques, des douleurs chroniques localisées dans le dos chez ces migrants. Mais il nous semble qu'il est imprudent, eu égard à la méthode utilisée et à l'effectif concerné, de conclure à une relation causale ou explicative entre le deuil d'un être cher chez ces réfugiés et les symptômes de somatisation. Néanmoins, les résultats observés ont permis à l'auteur de dire que ce lien est plausible, notamment à la lumière des résultats d'une étude pionnière (Kowalski et Bondmass, 2008). Ces derniers auteurs ont observé que les personnes en deuil manifestaient des symptômes somatiques pénibles tels que la sensation de serrement dans la gorge et dans la poitrine, des douleurs dorsales, cardiaques et musculo-squelettiques. II existe d'autres travaux empiriques qui ont trouvé un niveau très élevé de symptômes somatiques chez les immigrés, sans en faire le lien avec le deuil. Mentionnons au passage que dans une revue systématique, Rohlof et al. (2014) ont montré que les réfugiés non occidentaux étaient plus concernés par les symptômes somatiques que les réfugiés occidentaux.

Pour résumer cette section, il existe plusieurs travaux en psychopathologie et en psychiatrie transculturelle qui ont examiné le lien entre la santé mentale et les facteurs associés à l'immigration. Mais il en existe très peu qui ont cherché à élucider le lien entre la santé mentale et le deuil d'un parent ou d'un proche parent en contexte migratoire. L'étude de Hinton et al. (2013a) est l'une des rares études qui ont balisé la voie. Les résultats de cette étude suggèrent par ailleurs que les difficultés d'accéder aux ressources culturelles et religieuses du pays d'origine peuvent exacerber les réactions de deuil. Ce qui nous conduit à aborder quelques aspects anthropologiques et sociaux du deuil dans le contexte africain.

\section{Le deuil dans le contexte africain et spécificités en contexte migratoire}

Mis en perspective, les travaux de recherche que nous venons de présenter suggèrent que la perte d'un parent ou d'un proche parent "là-bas" dans le pays d'origine, ou "ici " dans le pays d'arrivée a sans doute un retentissent psychique déstabilisant. Elle est susceptible d'entraîner des réactions de deuil sévères et prolongées qui réinvestissent par association les traces mnésiques des pertes symboliques liées à l'expérience migratoire. De ce point de vue, les résultats observés par Mazzucato et al. (2006) semblent à maints égards éclairants. Dans cette étude menée auprès des migrants ghanéens aux Pays-Bas, les auteurs 
ont souligné que le deuil d'un parent ou d'un proche parent décédé dans le pays d'origine était ressenti comme une tragédie et vécu avec appréhension et inquiétude. Ces migrants étaient souvent les premiers à être informés du décès, à cause des espoirs placés sur eux en ce qui concerne l'organisation financière des funérailles. Ceux d'entre eux qui étaient dans l'impossibilité de participer aux cérémonies funéraires au pays d'origine avaient organisé des «bricolages rituels ${ }^{8}$ afin de tenter d'apaiser leur chagrin. Ces rituels renégociés consistaient notamment en une mini-veillée funèbre sans dépouilles mortelles du parent décédé, rythmée par des prières et des chants religieux pour le repos du défunt. À la suite de Thomas (1985), on peut penser que ces veillées sans corps du défunt ne permettent pas toujours une bonne séparation d'avec le mort. Elles laisseraient un sentiment d'inachevé fortement culpabilisant. Dans leur étude, Rachédi et al. (2016) ont fait deux suggestions intéressantes à partir des enjeux autour de la mort et du deuil chez les familles immigrantes au Québec. Ces auteures ont suggéré d'appréhender l'expérience du deuil chez les migrants non exclusivement sur le plan psychopathologique, mais aussi de prendre en compte l'implication des pratiques et savoirs cultuels (religieux ou non), souvent renégociés et transformés dans le cadre de l'immigration pour répondre au besoin et à la quête de sens à la mort d'un des leurs. Elles ont aussi suggéré de prendre en considération les réseaux d'entraide, au niveau local et/ou transnational qui joue un rôle de support social lors des événements de vie majeurs.

Dans les sociétés d'orientation traditionnelle, le deuil est une affaire du "collectif». II mobilise des solidarités actives autour des personnes directement touchées par la mort d'un proche. C'est la force de ces solidarités actives, selon la thèse durkheimienne, qui confère une efficacité symbolique et praxique aux rituels de deuil souvent accomplis pour contenir et réguler l'angoisse de la mort. Par définition, un rituel est "un ensemble d'actes formalisés, expressifs, porteurs d'une dimension symbolique. Il est caractérisé par une configuration spatiotemporelle spécifique, par le recours à une série d'objets, par des systèmes de comportements et de langages spécifiques, par des signes emblématiques dont le sens codé constitue l'un des biens d'un groupe" (Segalen, 1998). Les rituels de deuil sont un dispositif complexe institutionnalisé qui servent à faciliter le passage post-mortem du défunt et le processus du deuil des survivants (Thomas, 1985). Ils reposent sur des systèmes de représentations de la mort et du post-mortem qui peuvent varier d'un groupe social ou ethnique à un autre (Bonanno et Kaltman, 2001; Eisenbruch, 1984a et 1984b; Lobar et al., 2006; Panagiotopoulosa et al., 2013; Robben, 2004; Rosenblatt, 1993 et 2008). Ces variances transculturelles peuvent être résumées dans cette formule de Thomas (1975: 11) : "La mort biologique, fait de nature, se trouve constamment débordée par la mort, fait de culture». En France, des études (Aggoun, 2006; Chaïb, 2006) ont montré que, dans les minorités africaines, lorsqu'un décès a eu lieu dans le pays d'accueil, il est procédé au rite de rapatriement du corps ou des restes de la dépouille mortelle vers la terre des ancêtres, la terre du père (the Fatherland). Rapatrier le corps ou les restes de la dépouille mortelle du défunt et participer aux rituels de deuil sont d'impérieux devoirs de fidélité à la mémoire collective et d'affirmation de son identité culturelle (Aggoun, 2006; Chaïb, 2006; Thomas, 1985). Ces pratiques traduisent une nécessité de recourir

8 Cette expression est de Petit (2005). 
à des rituels de deuil pour contingenter, de manière symbolique, l'angoisse de la mort, et pour exprimer les derniers adieux au défunt. Dans plusieurs sociétés africaines, la présence des enfants, des petits-enfants et des proches du défunt au cours de ces rituels est un devoir de mémoire prescrit par les traditions. La pression familiale et sociale pour se conformer à ce devoir de mémoire est très accrue. Au Togo par exemple, habituellement, l'achat du cercueil et le cortège funèbre d'un défunt sont respectivement assurés par les fils et les petits-fils et revêtent des significations symboliques. C'est par ces actes que les fils et les petits-fils sont reconnus par la communauté comme ayant réussi à enterrer dignement leurs parents. Les veuves ou les veufs, les fils et les petits-fils sont les premiers endeuillés, soutenus ensuite par la communauté, qui préparent le voyage du défunt, du monde des êtres visibles vers le monde des ancêtres. Aussi ces conduites symboliques, assorties de bénédictions selon les croyances, vont-elles au-delà d'une piété familiale. Elles traduisent un contrat social avec la mémoire collective de la communauté. Le manquement à ces conduites peut générer des pathologies sur un fond de conflit culturel et identitaire (KokouKpolou et al., 2016). L'analyse de Rachédi et Tamouro (2012) est à juste titre fondée lorsqu'elles soulignent que la mort d'un parent "là-bas" dans le pays d'origine ou "ici» dans le pays d'accueil provoque des choix déchirants chez des migrants. Ils sont partagés entre les contraintes cultuelles et culturelles de "là-bas", mises à part les menaces politiques qui les ont fait fuir, et les réalités socioculturelles d' «ici ». Dans ce contexte, le vécu du deuil est problématique. II met à rude épreuve la santé mentale du migrant.

\section{Questions et objectifs de la recherche}

L'état actuel des connaissances sur le deuil consécutif à la mort d'un être cher en contexte migratoire et les problèmes psychopathologiques associés est moins avancé. II s'agit d'un sujet peu traité et qui pourtant présente des enjeux cliniques importants en termes de prise en charge des complications de deuil chez les populations migrantes. Quelles sont les réactions de deuil compliquées et persistantes qui font les spécificités du deuil en contexte migratoire et de quelles manières se manifestent-elles? Quels en sont les facteurs psychosociaux et culturels associés? Notre recherche avait pour but d'analyser les réactions de deuil ainsi que les risques de complications de celles-ci auprès d'immigrés togolais en France et en Belgique, en fonction de leurs caractéristiques sociodémographiques et de leur trajectoire migratoire (statut migratoire, durée de séjour dans le pays d'arrivée). De manière spécifique, il s'agit de :

1. Décrire les réactions de deuil compliquées ainsi que les symptômes associés chez ces immigrés;

2. Explorer la nature du lien entre les réactions de deuil, les symptômes dépressifs et les troubles somatiques;

3. Déterminer les facteurs de risques psychosociaux et culturels associés aux complications des réactions de deuil, aux symptômes dépressifs et troubles somatiques. 


\section{Méthode et matériels}

Notre démarche empirique a utilisé des méthodes mixtes : quantitative et qualitative. Dans un premier temps, nous avons collecté et analysé les données quantitatives. Afin d'approfondir ces données, nous avons mené, dans un deuxième temps, quatre entretiens exploratoires. Cette approche combinatoire est ce que Creswell et Plano Clark (2007) appellent une modélisation explicative.

\section{Participants et procédure}

Les participants à l'étude ont été recrutés à travers trois associations togolaises dont nous avons pris connaissance et dont les membres tiennent des rencontres régulières. Deux de ces associations sont localisées France et une en Belgique. Nous avons défini cinq critères de sélection. Tout d'abord, le décès devait concerner un parent ou un proche parent, car c'est ce type de deuil qui requiert essentiellement une implication rituelle (Eisenbruch, 1984b). Nous $\mathrm{n}^{\prime}$ avons pas pris en compte le deuil du conjoint ou le deuil d'un enfant dont la ritualisation en Afrique en général et au Togo en particulier est plus complexe. Ensuite, le décès devait remonter à six mois au moins de la date de l'enquête (Prigerson et al., 1995, 2009) ${ }^{9}$. Ensuite, les participants devaient être immigrés de première génération. Ce qui suppose qu'ils ne devaient pas être nés dans le pays d'immigration, d'un ou de parents immigré(s). En outre, ils devaient avoir résidé au moins douze mois dans le pays d'arrivée. Car, en général, il s'agit d'une période moyenne au terme de laquelle le statut de réfugié est régulièrement obtenu. Enfin, dernier critère, un consentement éclairé était nécessaire pour participer à l'étude. Pour ce faire, nous avons envoyé une lettre aux présidents des associations, présentant l'objectif et l'intérêt de l'étude. Les membres déclarés volontaires et ayant rempli les critères de sélection ci-dessus mentionnés ont constitué l'échantillon de l'étude. La passation des questionnaires et la collecte des données ont couvert une période de cinq mois, de juillet à novembre 2015. Au total, quatre-vingt-quatorze questionnaires ont été distribués dont quatre-vingt-deux ont été remplis et collectés. Nous en avons exclu huit qui comportaient plusieurs données manquantes. L'étude quantitative a donc inclus soixante-quatorze participants dont $62,2 \%$ étaient des hommes et $37,8 \%$ des femmes. L'âge moyen était de 37,12 ans $( \pm 11,03)$. Le volet qualitatif a porté sur quatre participants volontaires aux entretiens que nous avons proposés. II s'agit de deux femmes et deux hommes ( $M=44$ ans, $\pm 11,03)$, recrutés à travers les associations susmentionnées.

\section{Mesures}

S'agissant de l'étude quantitative, les participants ont rempli un questionnaire structuré en plusieurs sections dont six sont ci-dessous présentées selon la problématique abordée dans cet article.

9 Le critère de la durée varie d'un auteur à un autre. Selon les critères de diagnostic du deuil compliqué selon Prigerson et al. dont nous utilisons l'outil, cette durée est de six mois. 


\section{Caractéristiques sociodémographiques}

Elles regroupent le sexe, l'âge, le rang dans la fratrie (I'ainé, du deuxième à l'avant-dernier fils, le dernier fils) et la situation matrimoniale. Concernant le rang dans la fratrie, il revêt une signification culturelle particulière dans nombre de traditions africaines. Le rôle d'enterrer le parent défunt et de diriger la famille incombe à l'ainé de la fratrie. Selon Boucebci (1994), la position et les fonctions assignées aux ainés les prédisposent à une vulnérabilité psychopathologique qu'il décrit sous le concept de "syndrome d'aînité ".

\section{Informations sur l'immigration}

Les participants devaient renseigner leur pays d'accueil (France ou Belgique), leur statut d'immigration à l'arrivée, le principal motif d'immigration (études, regroupement familial, réfugié politique, réfugié de guerre), la durée de séjour dans le pays d'accueil.

\section{Caractéristiques et ritualité de la perte}

La nature du lien de parenté avec le défunt était indiquée (père, mère, grandpère/mère, tante/oncle, frère ou sœur) de même que le caractère prévisible du décès ( $1=$ mort prévisible, $2=$ mort inattendue/subite ou inopinée), la période du deuil (en mois). Il était aussi demandé aux participants des informations sur la ritualité funéraire (lieu du décès, lieu des cérémonies funéraires, rapatriement des dépouilles mortelles du défunt ou de ses restes au cas où le décès est survenu en pays d'immigration, participation ou non aux rituels, les motifs de non-participation).

\section{Réactions de deuil}

Pour examiner les réactions du deuil et en déterminer ses complications, nous avons utilisé l'inventaire du deuil compliqué de Prigerson et ses collègues (1995). Il existe plusieurs versions de cet outil : une première de dix-neuf items (Prigerson et al., 1995), une deuxième de trente-quatre items (Prigerson et Jacobs, 2001) et une troisième de dix-sept items. Cette dernière version a été validée en France (Gana et al., 2004; K'Delant, 2010). Cet outil mesure un seul construit sous-jacent au deuil compliqué (Boelen et Hoijtink, 2009). Les participants répondent à chaque item sur une échelle de Likert, de 0 à $4(0=$ presque jamais, $4=$ toujours, ou $0=$ pas de sentiment $d^{\prime}$ amertume, $4=$ un énorme sentiment d'amertume). Un item renseigne le niveau de perturbation du fonctionnement psychosocial. Pour présumer la présence du deuil compliqué, le score total doit être supérieur à 25, et les réactions du deuil doivent persister au-delà de six mois. Mais autant dans les contextes français que togolais, le seuil du deuil compliqué mesuré par cet outil est revu à 31 (Dassa et al., 2012; K'Delant, 2010). Précisons que pour les auteurs de cet article, ce seuil a une valeur indicative et non diagnostique. Dans cette étude, la consistance interne a été évaluée satisfaisante (Cronbach's $\alpha=.84$ ). L'analyse factorielle par la méthode rotation Oblimin directe indique que la structure de l'échelle repose sur un seul facteur. L'indice KMO est de .78, avec une signification du test de sphéricité de Bartlett $\left(\chi^{2}(\mathrm{ddl}=136)=439,68, p<.0001\right)$ montrant une bonne adéquation de notre échantillon $(N=74)$. Sur les dix-sept items de l'outil, seize saturent fortement sur un facteur (de .37 à .78), expliquant $31,2 \%$ de la variance totale. 
Le seul item dont le coefficient de saturation est faible est celui évaluant "le sentiment de nostalgie" (.19). Cet item qui semble moins relié au deuil de l'être aimé qu'au contexte migratoire n'a toutefois pas été supprimé, afin de réduire le biais qui serait lié au seuil de 31 .

Par ailleurs, à part les items de cet outil, nous avons ajouté deux questions dont la pertinence est ressortie de la pré-enquête. La première question évalue, sur une échelle de Likert de 0 à 4 ( 0 = presque jamais, $4=$ toujours), la présence des sentiments de culpabilité relatifs au contexte de la mort. La deuxième est une question ouverte, qui cherche à explorer les raisons de cette éventuelle culpabilité.

\section{Les symptômes dépressifs}

Nous avons utilisé l'échelle de dépression pour adulte-version courte (Clément et al., 2004) pour évaluer les symptômes dépressifs et le risque de dépression. Cette échelle comprend quatre items : les sentiments de tristesse, de vide, de sentir moins heureux et de désespoir. Les participants devaient répondre aux items par oui ou par non selon qu'ils ont vécu ces sentiments dans les sept jours précédant l'enquête. Plus le total des réponses affirmatives est élevé, plus on peut admettre l'hypothèse des risques d'une dépression.

\section{Les symptômes somatiques}

L'échelle de symptômes somatiques (The Somatic Symptom Scale-8, SSS-8) a été utilisée pour évaluer ces symptômes. II s'agit d'une version abrégée qui a été développée et validée par Gierk et al. (2014). L'échelle est composée de huit symptômes (problèmes gastriques [d'estomac ou d'intestin], douleur/mal de dos, douleur dans le bras, les jambes ou dans les articulations, maux de tête, vertiges, douleurs thoraciques ou essoufflement, sensation de fatigue ou de perte d'énergie et troubles de sommeil). Les participants répondent sur une échelle de Likert, de 0 à 4 ( $0=$ pas du tout, $4=$ beaucoup) en prenant en compte la manifestation de ces symptômes au cours des sept derniers jours précédant l'enquête. Plus le score est élevé, plus on présume la sévérité des symptômes. La consistance interne de l'échelle est solide (Cronbach's $\alpha=.89$ ).

Enfin, s'agissant de la méthode qualitative, nous avons construit un guide $\mathrm{d}^{\prime}$ entretien comportant quatre principaux thèmes couverts partiellement par les mesures quantitatives. D'abord, nous avons abordé avec les personnes enquêtées leur expérience migratoire. Ensuite, le deuxième thème a porté sur la qualité de la relation avec le parent défunt (exemples : la fréquence des communications téléphoniques; les formes de soutien apporté au parent dans ses derniers jours). Puis, le troisième thème a inclus plusieurs sous-thèmes : le contexte du décès, les réactions à l'annonce du décès, l'organisation et la participation aux obsèques et aux rituels de deuil. Enfin, le quatrième thème s'est focalisé sur l'adaptation à la perte (exemples: les ressources mobilisées pour surmonter le chagrin, le vécu de la perte, le sens donné à la perte). Les entretiens ont duré en moyenne quarante-deux minutes. 


\section{Plan d'analyse des données}

Les données quantitatives collectées ont été traitées à l'aide du logiciel SPSS 21. Nous avons effectué les statistiques descriptives pour déterminer la fréquence, la moyenne et l'écart-type des variables sociodémographiques, des variables reliées à l'immigration et les caractéristiques et la ritualité de la perte. Nous avons également effectué les Khi-deux lorsqu'il faut estimer le lien entre deux variables catégorielles (e.g., statut migratoire croisé au statut professionnel). Nous avons mené trois analyses de régression multiple (méthode pas-à-pas) afin de déterminer les facteurs associés aux trois variables dépendantes respectives de l'étude (réactions de deuil, risques de dépression et symptômes somatiques). Cette méthode a été privilégiée, car elle répond au caractère exploratoire de cette étude; elle permet en effet de rechercher les facteurs qui déterminent significativement la variable prédite, en éliminant ceux qui ne sont pas significatifs. De même, le poids de chaque variable explicative peut être directement évalué à partir de la variation du $R^{2}$. Pour répondre aux règles d'usage $d^{\prime} u n e$ analyse de régression linéaire, nous avons recodé certaines variables (e.g., rang dans la fratrie : 2 = ainés de fratrie, $1=$ autres rangs dans la fratrie; lien de parenté : 2 = père, mère, frère ou sœur, 1 = grand-parent, tante ou oncle; participation aux rituels : $2=$ oui, $1=$ non). Mentionnons que le taux de non-réponses à certaines échelles était inférieur à $4 \%$. Nous avons, dans ces cas, remplacé les données manquantes par la moyenne observée de la variable concernée sur l'ensemble de la population.

Comme indiqué plus haut, nous avons cherché à compléter et à approfondir les données statistiques grâce à la mobilisation des entretiens exploratoires. Nous avons choisi de présenter ces derniers sous forme d'études de cas afin de mettre en lumière la singularité de l'expérience de deuil de chaque enquêté.

\section{Résultats}

\section{Données statistiques}

\section{Profil des participants}

Le tableau 1 montre que notre échantillon avait une surreprésentation masculine $(62,2 \%$ étaient des hommes et $37,8 \%$ des femmes). L'âge moyen des immigrés et des réfugiés était respectivement de 33,9 ans $( \pm 10,01)$ et de 45,9 ans $( \pm 8,73)$. À l'arrivée en France ou en Belgique, $73 \%$ avaient le statut d'étudiant, de regroupement familial et $27 \%$ celui de réfugié (réfugié de guerre ou réfugié politique) ${ }^{10}$. Selon le rang dans la fratrie, quinze (soit $20,3 \%$ ) étaient des ainés de fratrie, dont douze hommes. On compte plus d'ainés de fratries réfugiés que d'autres immigrés ( $n=9 / 15$, soit $60 \%$ ). La période d'immigration dans I'ensemble était de 11,5 ans $( \pm 7,82): 43,2 \%$ avaient fait moins de dix ans et $56,8 \%$ plus de dix ans. Pour les réfugiés cette période était de 16,8 ans $( \pm 7,26)$, pour les autres immigrés elle était de 9,7 ans $( \pm 7,13)$. S'agissant du niveau d'études, $45,9 \%$ avaient un niveau universitaire et $54,1 \%$ un niveau d'études secondaires. Une différence très significative était observée entre le niveau de scolarisation

10 Après plusieurs mois voire plusieurs années. 
et le statut migratoire : $10 \%$ des réfugiés versus $59,3 \%$ des autres immigrés avaient le niveau d'études universitaires (Khi-deux $=14,26, p<.0001$ ). Quant au statut professionnel, $66,2 \%$ contre $33,8 \%$ exerçaient une activité professionnelle (à temps plein ou partiel). Nous avons observé un lien significatif entre le statut migratoire et le statut professionnel : $74,1 \%$ des migrants vs $45,5 \%$ des réfugiés avaient une occupation professionnelle (Khi-deux $=5,52, p<.05$ ). Selon la situation matrimoniale, 58,1\% étaient des célibataires, $41,9 \%$ vivaient en couple (mariés ou union libre). Lorsque le statut migratoire était croisé à la situation matrimoniale, on a noté que $85 \%$ des réfugiés (soit $n=17 / 20$ ) versus $25,9 \%$ des autres immigrés (soit $n=14 / 54$ ) vivaient en couple (Khi-deux $=20,92, p<.0001$ ).

Tableau 1 : Données descriptives relatives au profil sociodémographique des participants

\begin{tabular}{|c|c|c|c|}
\hline Variables & $\mathbf{N}$ & $\%$ & $\begin{array}{c}\text { Moyenne } \\
\text { (Écart-type) }\end{array}$ \\
\hline \multicolumn{4}{|l|}{ Variables sociodémographiques } \\
\hline \multicolumn{4}{|l|}{ Sexe } \\
\hline Masculin & 46 & 62,2 & \\
\hline Féminin & 28 & 37,8 & \\
\hline \multicolumn{4}{|l|}{$\hat{A} g e$} \\
\hline $20-29$ ans & 34 & 45,9 & $37,12(11,03)$ \\
\hline $30-49$ ans & 24 & 32,4 & \\
\hline 50 ans et plus & 16 & 21,6 & \\
\hline \multicolumn{4}{|l|}{ Rang dans la fratrie } \\
\hline Ainé & 15 & 20,3 & \\
\hline Deuxième à avant-dernier & 48 & 64,9 & \\
\hline Dernier enfant & 11 & 14,9 & \\
\hline \multicolumn{4}{|l|}{ Niveau d'études } \\
\hline Collège, lycée & 40 & 54,1 & \\
\hline Université & 34 & 45,9 & \\
\hline \multicolumn{4}{|l|}{ Situation familiale } \\
\hline Célibataire & 43 & 58,1 & \\
\hline En couple & 31 & 41,9 & \\
\hline \multicolumn{4}{|l|}{ Statut professionnel } \\
\hline Travail à temps plein ou partiel & 49 & 66,2 & \\
\hline Chômage & 25 & 33,8 & \\
\hline \multicolumn{4}{|l|}{ Trajectoire migratoire } \\
\hline \multicolumn{4}{|l|}{ Statut migratoire } \\
\hline Réfugié & 20 & 27,0 & \\
\hline Autres immigrés & 54 & 73,0 & \\
\hline \multicolumn{4}{|l|}{ Durée d'immigration } \\
\hline Moins de 10 ans & 32 & 43,2 & $11,47(7,82)$ \\
\hline Plus de 10 ans & 42 & 56,8 & \\
\hline
\end{tabular}

Source : Kokou-Kpolou et al., données de terrain, juillet à novembre 2015.

\section{Caractéristiques et ritualité de la perte}

Les résultats du tableau 2 indiquent que dans $46 \%$ des cas, le décès avait concerné un des parents (le père ou la mère), dans $24,3 \%$ il avait concerné un grand-parent, dans $17,6 \%$ un oncle, une tante et dans $12,2 \%$ un frère ou une sœur. La période moyenne du deuil était de 47,01 mois $( \pm 34,76): 51,4 \%$ 
des décès ont eu lieu il y a moins de 36 mois et $48,6 \%$ plus de 36 mois. Respectivement dans $59,5 \%$ et $35,1 \%$ des cas, le décès est survenu au pays d'origine (au Togo) et au pays d'accueil (France ou Belgique). Deux décès sont survenus aux États-Unis, un au Ghana et un en Côte d'Ivoire. Dans les cas où le décès est survenu à l'étranger (trente cas), les rituels funéraires s'y sont déroulés dans une proportion de $73,3 \%(n=22)$. Dans de tels cas, il a été procédé dans $54,5 \%$ cas $(n=12 / 22)$ au transfert des restes du défunt vers le pays d'origine. Dans huit cas le corps du défunt a été rapatrié.

Dans I'ensemble, $35,1 \%$ contre $64,9 \%$ des sujets avaient participé aux rituels de deuil. Une précision importante : seulement trois ainés sur les quinze ont pu participer aux rituels funéraires de leur parent. Dans les cas où le décès a eu lieu au pays d'origine, les difficultés administratives et/ou financières ont été les motifs majoritairement évoqués pour justifier la non-participation aux rituels de deuil.

Tableau 2 : Données descriptives relatives aux caractéristiques de la perte et à la participation aux rituels funéraires

\begin{tabular}{llcc}
\hline Caractéristiques de la perte & N & $\%$ & $\begin{array}{c}\text { Moyenne } \\
\text { (Écart-type) }\end{array}$ \\
\hline Lien de parenté & 19 & 25,7 \\
Père & 15 & 20,3 \\
Mère & 18 & 24,3 \\
Grand-père/mère & 13 & 17,6 \\
Oncle, tante & 09 & 12,2 \\
Frère, sœur & & \\
Caractère prévisible de la mort & 49 & 66,2 \\
Mort attendue & 25 & 33,8 \\
Mort non attendue & & 59,5 \\
\hline Lieu où est survenu le décès & 44 & 35,1 \\
Pays d'origine (Togo) & 26 & 05,4 \\
Pays d'arrivée (France ou Belgique) & 04 & 51,4 \\
Autres pays & & 48,6 \\
\hline Durée du deuil & 38 & 35,01 (34,76) \\
Moins de 3 ans & 36 & 64,9 \\
Plus de 3 ans & & \\
\hline Participation aux rituels & 26 & 48 & \\
Oui & & \\
Non & & \\
\hline
\end{tabular}

Source : Kokou-Kpolou et al., données de terrain, juillet à novembre 2015.

\section{Réactions de deuil persistantes, symptômes dépressifs et somatiques}

Au seuil pathologique $\geq 31$ (Dassa et al., 2012; K'Delant, 2010) avec la persistance des réactions de deuil au-delà de six mois (Prigerson et al., 1995), $41,9 \%$ des immigrés et des réfugiés de notre échantillon auraient présenté un deuil compliqué. Le score moyen à I'IDC était de $34,58( \pm 8,31)$. Les réactions de deuil sévères et persistantes étaient l'hébétude ou le choc $(44,6 \%)$, la nostalgie $(41,9 \%)$, la solitude $(35,1 \%)$, I'accablement par la douleur $(32,5 \%)$, etc. On a noté une recrudescence du sentiment de culpabilité $(47,3 \%)$ dont les raisons 
évoquées étaient l'impossibilité d'une part d'avoir participé aux cérémonies funéraires et d'autre part d'avoir apporté assistance financière et matérielle suffisante au parent ou au proche parent avant son décès. Concernant la dépression, trois symptômes étaient dominants : le sentiment de vide $(68,9 \%)$, la tristesse $(51,4 \%)$ et le sentiment de sentir moins heureux $(41,9 \%)$. Le désespoir était moins rapporté $(21,6 \%)$. S'agissant des symptômes somatiques, les douleurs articulaires $(45,9 \%)$, les douleurs thoraciques $(40,5 \%)$, les troubles de sommeil $(37,8 \%)$, les maux de tête, les vertiges étaient plus souvent rapportés.

\section{Facteurs de risque associés aux complications de deuil, aux symptômes dépressifs et somatiques}

Le tableau 3 présente les résultats à partir de l'analyse de régression linéaire (méthode pas-à-pas). II s'est agi d'identifier les facteurs associés aux complications du deuil, aux risques de dépression et aux symptômes somatiques.

\section{Facteurs sociodémographiques}

Deux facteurs sociodémographiques sont communs aux complications de deuil, aux risques de dépression et aux symptômes somatiques. II s'agit du statut professionnel et de la situation matrimoniale. Le premier explique à lui seul $35,1 \%$ de la variance des complications du deuil. Les immigrés et les réfugiés qui n'exerçaient pas d'activité professionnelle et ceux qui vivaient en couple avaient plus manifesté des complications de réaction de deuil $(B=$ $0,439, p<.0001)$. Le deuxième facteur prédit $31,8 \%$ de variance des risques de dépression et $18,8 \%$ de la variance des symptômes somatiques. On observe que ce sont les immigrés et les réfugiés qui vivaient en couple qui avaient plus manifesté ces risques de dépression $(B=0,332, p<.01$ ) et de symptômes somatiques $(\beta=0,302, p<.01)$. Par ailleurs, les ainés de fratrie étaient plus vulnérables aux complications de deuil $(\beta=0,209, p<.05)$ et les personnes plus âgées plus enclines aux risques de dépression $(\beta=0,290, p<.05)$.

\section{Facteurs associés à l'immigration}

Le statut d'immigration est très fortement associé aux variables dépendantes. Les réfugiés avaient plus manifesté des réactions de deuil compliquées ( $\beta=0,521, p<.0001)$, des risques de dépression $(\beta=0,443, p<.0001)$ et des symptômes somatiques ( $\beta=0,523, p<.0001$ ). Plus la période d'immigration est longue, plus les réactions de deuil étaient compliquées $(B=0,282, p<.01$ ) et les risques de dépression accrus $(B=0,315, p<.01)$. Concernant la durée de séjour, le point de césure montre qu'à partir de dix ans, ces troubles seraient plus compliqués.

Caractéristiques et ritualité de la perte

Lorsque le défunt est un parent ou un frère ou une sœur, les réactions de deuil $(B=0,526, p<.0001)$, les risques de dépression $(B=0,363, p<.01)$ et les symptômes somatiques $(\beta=0,383, p<.0001)$ sont plus exacerbés. La participation aux cérémonies funéraires est associée à moins de risques de complication de deuil $(\beta=-0,270, p<.01)$ et à moins de risques de manifester les symptômes somatiques $(\beta=-0,402, p<.01)$. Elle ne prédit pas les risques de dépression. 
Relation entre la culpabilité, le deuil, les risques de dépression et les symptômes somatiques

Les réactions de deuil sont très fortement associées aux risques de dépression $(\beta=0,759, p<.0001)$. Dans ce modèle de régression, les risques de dépression ne sont pas associés aux symptômes somatiques. En revanche, la culpabilité et l'intensité des réactions de deuil expliquent environ $55 \%$ de la variance des symptômes somatiques.

\section{Tableau 3 : Analyse de régression linéaire multiple (méthode pas-à-pas) des facteurs associés aux complications du deuil, aux risques de dépression et aux symptômes somatiques}

\begin{tabular}{|c|c|c|c|c|c|c|}
\hline \multirow[t]{2}{*}{ Prédicteurs } & \multicolumn{2}{|c|}{ Réactions de deuil } & \multicolumn{2}{|c|}{$\begin{array}{c}\text { Symptômes } \\
\text { dépressifs }\end{array}$} & \multicolumn{2}{|c|}{$\begin{array}{l}\text { Symptômes } \\
\text { somatiques }\end{array}$} \\
\hline & R2adj & $F$ & R2adj & $F$ & R2adj & $F$ \\
\hline \multicolumn{7}{|l|}{ Données sociodémographiques } \\
\hline Sexe & ns & ns & ns & ns & ns & ns \\
\hline Âge & ns & ns & 0,075 & $9,91 * *$ & ns & ns \\
\hline Rang dans la fratrie & 0,029 & $4,97^{*}$ & ns & ns & ns & ns \\
\hline Statut professionnel & 0,351 & $40,45^{* * *}$ & 0,030 & $4,72^{*}$ & 0,068 & $5,65^{*}$ \\
\hline Statut matrimonial & 0,087 & $12,11 * *$ & 0,318 & $35,00 * * *$ & 0,138 & $12,66 * * *$ \\
\hline \multicolumn{7}{|l|}{ Trajectoire migratoire } \\
\hline Statut migratoire & 0,398 & $49,35 * * *$ & 0,320 & $35,32 * * *$ & 0,264 & $27,17 * * *$ \\
\hline Durée de séjour/pays d'accueil & 0,060 & $8,86^{* *}$ & 0,075 & $9,95 * *$ & ns & ns \\
\hline \multicolumn{7}{|c|}{ Caractéristiques et ritualité de la perte } \\
\hline Lien de parenté & 0,236 & $23,53^{* * *}$ & 0,120 & $10,92 * * *$ & 0,136 & $14,18^{* * *}$ \\
\hline Type de mort & ns & ns & ns & ns & ns & ns \\
\hline Période de deuil & ns & ns & ns & ns & ns & ns \\
\hline Participation aux rituels & 0,063 & $7,48 * *$ & ns & ns & 0,118 & $10,75^{* * *}$ \\
\hline \multicolumn{7}{|l|}{ Variables dépendantes } \\
\hline Culpabilité & 0,118 & $10,79 * *$ & ns & ns & 0,167 & $27,71 * * *$ \\
\hline Réactions de deuil & - & - & 0,571 & $97,99 * * *$ & 0,382 & $46,11 * * *$ \\
\hline Dépression & - & - & - & - & ns & ns \\
\hline
\end{tabular}

ns $=$ non significatif; ${ }^{*} p<.05 ;{ }^{* *} p<.01 ;{ }^{* * *} p<.0001$.

Source : Kokou-Kpolou et al., données de terrain, juillet à novembre 2015.

\section{Données qualitatives}

Les quatre études de cas élargissent le regard du lecteur et offrent une lecture plurielle et riche de l'expérience du deuil en situation migratoire. Plusieurs axes d'analyse s'y dégagent et nous en avons retenu quatre en relation directe avec notre problématique et les variables associées (les réactions de deuil et symptômes associés en fonction du lien avec le parent défunt, du statut migratoire, de l'identité biculturelle des immigrés et du statut professionnel).

En tout premier lieu, la qualité du lien avec le parent conditionne en partie la réaction de choc ou d'hébétude intense manifestée par la plupart des participants. En général, la littérature du deuil montre que lorsque la perte est prévisible, elle s'accompagne moins de la réaction de choc. Dans ces études de 
cas, certes moins représentatives de la diversité des expériences que l'on peut rencontrer, il en ressort que malgré l'âge du défunt et le caractère prévisible et imminent de la perte, les participants ont réagi à la perte par un sentiment de choc. Cela peut suggérer que l'expérience migratoire ne permet pas de se préparer à la perte même lorsqu'elle est prévisible. Cette hypothèse est à relier d'une part à la distance géographique qui ne leur permet pas de se confronter aux éléments matériels de la réalité irréversible de la perte et d'autre part au manque du soutien social non disponible ou immédiat qui amplifierait l'effet du choc lié à l'annonce du décès.

En deuxième lieu, nous observons une sévérité du deuil chez les réfugiés politiques. En général, lorsque le deuil survient dans le pays d'origine, ceux-ci sont dans une impossibilité de retour. Leur retour les expose à des menaces qui planent sur leur vie. Sous un autre angle, la sévérité du deuil est également à mettre en lien, tout au moins en partie, avec la situation d'immigration irrégulière (à l'arrivée dans le pays d'installation de certains immigrés. Ce qui, à l'évidence, ne favorise pas leur intégration notamment économique. Confrontés au deuil d'un parent, ils sont démunis de moyens financiers pour satisfaire aux obligations et aux attentes parfois démesurées qui sont placées sur eux. Ce qui souvent génère et exacerbe le sentiment de culpabilité manifesté par certains participants.

En troisième lieu, il ressort des études de cas présentées que le niveau d'intégration de la culture d'origine et de la culture d'accueil - intégration biculturelle - intervient dans l'élaboration du deuil. Lorsque l'immigré investit les croyances culturelles du pays d'origine avec une difficulté de décentrage et de renégociation de celles-ci, l'adaptation à la perte parait plus problématique sur fond de conflits identitaires et de loyauté.

En quatrième lieu, nous observons que face aux contraintes professionnelles et administratives, certains immigrés choisissent à travers un mécanisme de refoulement partiel de ne pas exprimer les réactions de deuil. Le deuil est temporairement gelé. Selon la terminologie courante, on parle du deuil différé. Les manifestations du deuil ressurgissent plus tard dans d'autres circonstances de manière inattendue, accompagnées de perturbations émotionnelles et parfois somatiques intenses.

\section{Étude de cas 1 : Madame AA}

Madame AA est âgée de trente-huit ans, en couple, mère d'un enfant. Elle a immigré en Belgique, il y a onze ans. L'annonce du décès de son père, en 2015, a été vécue comme un choc violent. "C'était un matin vers 11 heures, je me suis effondrée par terre et j'ai perdu connaissance pendant plusieurs minutes [confie-t-elle]. La veille [renchérit-elle], j'ai parlé avec mon père au téléphone et il ne s'était plaint d'aucun souci de santé». Par manque de moyens financiers, madame AA n'a pas pu participer aux obsèques de son père qui ont eu lieu deux mois après le décès. Elle s'explique : "Nous avions une situation difficile. J'avais un enfant de dix-huit mois environ. Mon partenaire et moi étions au chômage". Les yeux embués de larmes, elle dit que son père était tout le bien qui lui restait. Puis elle confie : "Nous avons dû nous endetter pour acheter le cercueil». Madame AA fait le projet d'aller au Togo en 2020, en vue de participer 
aux deuxièmes funérailles de son père. "C'est seulement en ce moment-là, dit-elle, lorsque j'aurai visité sa tombe que je pourrai être apaisée. Pour l'instant, je ne saurais dire si j'ai fait le deuil».

\section{Étude de cas 2 : Monsieur BB}

Monsieur BB, soixante-deux ans, est un ancien haut cadre d'administration, accueilli en France en 2010 comme réfugié politique. Son âge ne lui permet pas une reconversion professionnelle aisée. II décrit ses conditions de vie financières comme précaires, réduites à des minima sociaux. Lorsque sa mère est décédée en 2013, il ne pouvait pas se rendre à ses obsèques compte tenu de son statut, car il encourait le risque d'être incarcéré s'il foulait le sol du territoire national. Monsieur BB s'est donc contenté d'organiser une veillée de prière en la mémoire de sa défunte mère. La veillée de prière a été rendue possible grâce à la participation de quelques amis et membres de sa communauté d'origine. Par solidarité, ces derniers lui ont fait des dons en argent. C'est cet argent qu'il dit avoir envoyé à la famille restée au pays, afin de contribuer à organisation des obsèques de sa mère. Tout au long de l'entretien, monsieur BB manifestait un sentiment de culpabilité. Pour lui, en tant qu'ainé de la famille, il est impensable et impardonnable qu'il ne soit pas physiquement présent aux obsèques de sa mère. Monsieur BB attribue la cause des difficultés existentielles actuelles qu'il rencontre à une punition infligée par les ancêtres pour n'avoir été à la hauteur des attentes placées sur un ainé de famille.

\section{Étude de cas 3 : Madame CC}

Madame CC, âgée de trente-cinq ans, est le quatrième d'une fratrie de cinq. Elle vit en couple depuis un an. Elle a perdu sa mère en 2012, alors qu'elle finissait sa formation paramédicale. Sa mère est décédée en France, où elle venait régulièrement leur rendre visite. À soixante-quatre ans, elle serait décédée des suites d'un infarctus. Elle fut enterrée en France et un an après, les restes du corps ont été rapatriés au Togo pour les dernières cérémonies. Madame CC parle du vécu de la mort de sa mère en ces termes : "C'était la ruine de ma vie; c'est comme si ma vie ici n'avait plus de sens". Au cours des cérémonies du deuil et d'inhumation, elle dit s'être sentie entourée et soutenue par la présence de ses frères qui vivent en France et en Allemagne. Mais très vite, elle avait tenté d'oublier cet événement en se concentrant sur la fin de sa formation. C'est plus tard, dans $\mathrm{d}^{\prime}$ autres circonstances qu'elle se serait sentie affectée par la mort de sa mère. D'abord, après la réussite à son examen de fin d'études, madame CC dit avoir été envahie sans cause apparente par des sentiments de colère et de tristesse pendant cinq mois; et elle tentait de rechercher sa mère (elle lui arrivait de téléphoner au numéro de sa mère, ou de demander à ses frères si celle-ci viendrait les visiter). Elle faisait des cauchemars répétés et au réveil, elle se sentait mentalement épuisée. Elle dit avoir suivi un traitement médical pour motif de troubles de sommeil. Puis environ deux ans plus tard, alors qu'elle connut sa première maternité, les souvenirs de sa mère étaient vivaces et insupportables. Dans le même temps, elle était constamment angoissée à l'idée d'oublier sa mère. Pour tenter d'apaiser son angoisse, elle confie avoir donné à sa fille le nom de sa mère. 


\section{Étude de cas 4 : Monsieur DD}

Monsieur DD est âgé de quarante-et-un ans. Ingénieur des ponts et chaussées, il vit en France depuis vingt ans. Son père est décédé aux États-Unis où il suivait, une fois par an, des soins intensifs pour une maladie chronique. Les obsèques ont été organisées sur place. Monsieur DD n'a pas participé en personne aux obsèques, mais confie s'être impliqué dans les dépenses des soins puis des obsèques de son père : "Au début, j'ai eu du mal à accepter sa mort, mais après je me sens fier pour tout ce que j'ai pu lui faire. Là où il est, je suis certain qu'il est aussi fier de moi, et je sais qu'il veille sur moi. Je le vois parfois dans mes rêves». Monsieur DD dit ne pas s'empresser pour le rapatriement des restes du corps de son père malgré la pression de ses oncles paternels. II a fait faire une vidéo du déroulement des obsèques; lorsque les souvenirs de son père semblent moins présents, il visualise la vidéo et se console.

\section{Discussion}

L'objectif principal poursuivi par cette étude transversale était d'examiner les réactions de deuil ainsi que les risques de complications de ces réactions dans la population d'immigrés et réfugiés ouest-africains en Europe, en fonction de leur profil sociodémographique et migratoire (statut migratoire, durée de séjour dans le pays d'accueil). Les résultats auxquels nous sommes parvenus indiquent que l'expérience du deuil d'un parent proche en contexte migratoire, que ce soit "ici", dans le pays d'accueil, ou "là-bas", dans le pays d'origine ou encore ailleurs, revêt une charge traumatogène et psychopathogène.

\section{Incidence des complications du deuil en contexte migratoire}

Dans notre étude, la prévalence du deuil compliqué était de 41,9\%. Cette prévalence, qu'il faut avec parcimonie considérer comme indicative, est largement supérieure à celle estimée dans la population générale qui varie de 10 à 25,4 \% (Lundorff et al., 2017; Newson et al., 2011). Elle se rapproche logiquement de celle de $54 \%$ trouvée par Craig et al. (2008) dans un échantillon de 126 réfugiés bosniaques diagnostiqués et suivis pour trouble de stress posttraumatique. II doit être mentionné que dans la présente étude le type de lien avec le défunt était le lien de parenté. Car, comme l'a si bien souligné Eisenbruch (1984b : 325) "seule la mort d'un parent a des conséquences sociales immédiates, puisque les personnes en deuil doivent assumer un ensemble d'obligations sociales comme prescrit par son groupe ethnique ${ }^{11}$ ". Ce qui explique en partie la sévérité des réactions de deuil, caractérisée par le choc, la douleur, etc. Nos résultats ont montré un lien très fort entre le deuil et les risques de dépression. Le lien entre le deuil et la dépression dans la population générale est abondamment documenté (Bonanno et al., 2007; Zisook et al., 2010). Dans la population immigrante particulièrement, la dépression est surtout associée aux difficultés d'intégration (Craig et al., 2008; Levecque et Van Rossem, 2015; Missinne et Bracke, 2012). Dans la présente étude, nous n'avons pas évalué les difficultés d'intégration des immigrés, mais les résultats de l'étude qualitative suggèrent que les symptômes dépressifs provoqués par la sévérité du deuil

11 Traduction par les auteurs. 
pourraient s'expliquer aussi par les difficultés à donner sens à son expérience migratoire lorsque la mort frappe les personnes qui se sont impliquées dans son projet migratoire.

\section{Lien entre deuil, dépression et troubles somatiques en contexte migratoire}

Nous estimons par ailleurs que nos résultats apportent un regard nouveau sur le lien entre le deuil, la dépression et les symptômes somatiques. En effet, l'analyse de régression a permis de montrer que les complications du deuil expliquent les risques de dépression. Ce que nous venons d'élucider. Les résultats ont montré aussi que ce n'est pas la souffrance dépressive qui explique les symptômes somatiques, mais plutôt les complications de deuil et le sentiment de culpabilité. Notre résultat donne raison à l'étude de Sanz (2012) qui a tenté d'éclairer le lien entre le deuil et la somatisation chez des immigrés bosniaques et albanais. Sanz a observé chez ces derniers des plaintes somatiques, des douleurs chroniques localisées dans le dos. Dans la présente étude, ce sont les douleurs articulaires, les douleurs thoraciques et les troubles de sommeil qui viennent en tête des symptômes somatiques. Pour Sanz (2012 : 82), l'impact du deuil dans l'émergence et la chronicisation des symptômes somatiques n'est pas à dissocier d'autres facteurs comme la situation économique des immigrés, les tragédies liées à leur passé migratoire, etc. La somatisation est au carrefour de tous ces facteurs, dont le deuil vient exacerber l'expression. Nos résultats vont dans la même direction que ceux observés par Ritsner et al. (2000) auprès des migrants de l'ex-Union soviétique installés en Israël. Ces auteurs ont relevé que plus l'indice de la détresse psychologique était élevé, plus le nombre de symptômes somatiques l'était aussi. Ceci nous autorise à penser comme Adansikou et al. (2017) que lorsque la détresse accumulée est importante et dépasse le seuil d'adaptation des capacités psychiques, le corps en devient le réceptacle à travers plusieurs modalités expressives.

\section{Facteurs psychosociaux associés aux complications du deuil}

Nos résultats ont montré que le statut professionnel est un facteur très significatif, associé aux complications du deuil, aux risques de dépression et aux symptômes somatiques. Ces résultats vont dans le droit-fil des études ayant observé le lien entre le statut professionnel et la dépression chez les immigrés de première génération en Europe (Levecque et Van Rossem, 2015; Missinne et Bracke, 2012). De même, ils confirment les résultats de Fenta et al. (2010) qui ont trouvé un lien très significatif entre le statut professionnel et les symptômes somatiques chez les immigrés éthiopiens à Toronto au Canada. En situation migratoire, l'occupation professionnelle est l'un des indicateurs d'intégration et d'insertion sociale d'une grande importance. Elle constitue, sur le plan psychosocial, un facteur protecteur des stress liés à l'immigration. Elle permet $\mathrm{d}^{\prime}$ apporter une assistance matérielle et financière aux parents, là-bas dans le pays d'origine. En effet, les immigrés dont l'intégration dans le pays d'accueil, se fait avec difficultés, pour des motifs administratifs, pour manque de moyens financiers, et qui sont dans l'impossibilité de se rendre aux obsèques du défunt, éprouvent un chagrin très intense (Mazzucato et al., 2006). Ces résultats mettent ainsi en évidence le poids des conditions socioéconomiques en contexte migratoire sur les réactions du deuil et la santé mentale. Lorsque la mort du parent 
survient dans le pays d'origine, ce qui est majoritairement le cas dans notre étude, les raisons de non-participation aux rituels funéraires évoquées étaient les conditions socioéconomiques et administratives (problèmes de papiers). Cette impossibilité de retour exacerbe le sentiment d'accablement par la perte. Une autre raison à prendre en considération est l'insécurité ressentie surtout chez les réfugiés politiques qui avaient peur de s'exposer aux menaces s'ils retournaient au pays d'origine dans le cadre des rituels funéraires de leur parent défunt. Ce qui atteste, comme I'ont souligné Rachédi et Tamouro (2012), à quel point les immigrés endeuillés sont confrontés à des choix déchirants susceptibles de provoquer une décompensation psychique.

Notre étude a révélé que le deuil compliqué afflige davantage les migrants vivant en couple. A priori ce résultat parait surprenant, car dans la population endeuillée le deuil compliqué, à part le deuil conjugal et le deuil périnatal, est moins associé au statut matrimonial (Fujisawa et al., 2010; Newson et al., 2011). La vie en couple est en général considérée comme un facteur protecteur. Au-delà du fait que nous avons observé que dans notre échantillon plus de réfugiés vivaient en couple que les autres immigrés, ce qui peut aussi s'expliquer par la différence d'âge entre ces deux groupes, nous pensons que la parentalité actuelle réactiverait les sentiments d'une dette envers ses propres parents. $C^{\prime}$ est ce qu'illustre le cas de madame AA. II s'agirait là d'un aspect culturel à prendre en considération. De même, nous avons trouvé que les ainés de fratrie étaient plus vulnérables aux complications de réactions deuil d'autant plus que dans les représentations collectives. Le poids de leur hiérarchie et de leur rôle dans le roman familial constitue un haut risque de vulnérabilité psychopathologique (Boucebci, 1994). En effet, leur absence physique lors des cérémonies funéraires ( $n=12 / 15$ des ainés n'ont pas participé aux rituels), même s'ils ont contribué financièrement à l'organisation de celles-ci, est souvent vécue par eux-mêmes comme une défaillance vis-à-vis de leur communauté. L'étude de Moukouta et al. (2016 : 2) soutient notre argumentation. Les auteurs ont montré à travers un des deux cas qui fondent leur étude, comment l'absence aux obsèques de sa mère au Congo est vécue douloureusement par un patient "dépressif ", ainé de sa fratrie et réfugié politique en France. Pour ce dernier, cette absence n'est pardonnable ni par les membres de sa famille ni par les ancêtres, car il a failli aux attentes du groupe. Dans notre étude, c'est le cas par exemple de monsieur BB qui culpabilise pour avoir manqué à ses obligations d'ainé et vit sa détresse comme la conséquence d'un châtiment infligé par ses ancêtres. L'impossibilité de retour évoquée plus haut, ajoutée à l'indisponibilité des supports rituels, exacerbe les sentiments de douleur et de culpabilité. D'après Eisenbruch (1984b), la recrudescence de la culpabilité est liée à l'incapacité ou l'impossibilité d'avoir comblé les attentes du défunt : par exemple, porter suffisamment une assistante financière à son parent avant sa mort. Cette culpabilité est parfois suivie de tentatives de réparation symbolique ou réelle : I'investissement financier dans les funérailles (payer le cercueil même s'il faut s'endetter), traduisant a posteriori le besoin d'affirmation de son lien au groupe ethnique d'appartenance (Mazzucato et al., 2006).

Notre étude a également apporté une preuve empirique des bénéfices psychologiques des rituels de deuil dans le processus d'adaptation à la perte d'un être cher. Lorsque les facteurs sociodémographiques sont contrôlés, nos résultats ont montré que les immigrés endeuillés qui ont participé aux rituels de 
deuil avaient manifesté des réactions de deuil moins intenses. Les résultats ont révélé également qu'ils ont rapporté des sentiments de culpabilité moins accablants que ceux qui n'ont pas pu participer aux rituels de deuil. Dans l'ensemble, ces résultats vont le sens des travaux de Thomas (1985) qui soutiennent que les rituels de deuil d'une part fournissent un espace d'expression et de régulation des émotions et d'autre part remplissent, au plan imaginaire, une haute fonction d'expiation des sentiments de culpabilité.

\section{Conclusion et perspectives}

En définitive, les résultats de cette étude posent les prémisses à des études ultérieures sur le deuil en contexte migratoire et ouvrent des pistes de réflexion sur les dispositifs de prise en charge du deuil compliqué chez les immigrés (Craig et al., 2008). Les résultats pourraient permettre un affinement dans la compréhension heuristique du deuil d'un parent ou d'un proche parent ainsi que des troubles associés (dépression, symptômes somatiques) chez les immigrés selon leur profil sociodémographique et migratoire.

Nous voulons attirer l'attention des lecteurs sur la généralisation de la portée de ces résultats qui doit se faire avec précaution. Bien que basée sur une méthodologie mixte qui a permis de saisir plusieurs aspects de la problématique soulevée, notre étude comporte des limites que des études à venir, nous l'espérons, pourront améliorer. D'abord, notre échantillon est moins représentatif (cinquante-quatre migrants vs vingt réfugiés), ce qui ne permet pas de prétendre à une généralisation des résultats. Une perspective intéressante serait d'entreprendre, sur la base d'un échantillon représentatif, une approche comparative rigoureuse selon le statut d'immigration. Par ailleurs, les résultats de notre étude qualitative suggèrent qu'en abordant la variable statut d'immigration il faut prendre en compte la situation d'immigration régulière ou non, car elle conditionne le niveau d'intégration socioculturelle et économique dans le pays d'accueil. Pour améliorer notre approche méthodologique, on peut envisager étude comparative entre les immigrés d'une part et une population locale d'origine qui sert de groupe témoin d'autre part. En outre, des facteurs comme les traumatismes pré-migratoires ( $\mathrm{Li}, 2016)$, les conditions d'installation (Craig et al., 2008), les conditions socioéconomiques (type d'emploi, niveau de revenu mensuel, etc.) n'ont pas été pris en compte. De même, les pertes antérieures ou cumulées n'ont pas été intégrées dans cette étude. Vu sous cet angle, un protocole d'étude plus global devra être envisagé en vue d'identifier les liens concomitants entre plusieurs facteurs et leur influence sur les réactions de deuil. II serait aussi pertinent d'intégrer à un tel protocole des instruments de mesure permettant d'objectiver la qualité de vie ou la santé globale des immigrés (Crollard et al., 2012). 


\section{Références bibliographiques}

Adansikou Kouami, Kokou-Kpolou Cyrille Kossigan, Mbassa Menick Daniel et Moukouta Charlemagne S. (2017) Influence de la religion dans la somatisation dépressive en Afrique, Annales Médico-Psychologiques, 175 (6), pp. 536-540.

Aggoun Atmane (2006) Les musulmans face à la mort en France, Paris, Vuibert, $156 \mathrm{p}$.

Améganvi Claude (2011) Livre Noir contre l'impunité au Togo : 13 janvier 1963-13 janvier 2010: 47 ans d'assassinats et d'ímpunité au Togo sous la dictature sanglante du clan des Gnassingbé, Lomé, Editions Nyamwo, 320 p.

American Psychiatric Association (2013) Diagnostic and Statistical Manual of Mental Disorders (DSM-5), Washington, DC, American Psychiatric Association.

Badji Bougoul (1993) La folie en Afrique. Une rivalité pathologique, Paris, L'Harmattan, $211 \mathrm{p}$.

Bass Loretta E. (2014) African immigrant families in another France, University of Oklahoma, Palgrave Macmillan, $186 \mathrm{p}$.

Baubet Thierry et Moro Marie Rose (2013) Effets de la migration et de I'exil, in Thierry Baubet et Marie Rose Moro Éds., Psychopathologie transculturelle, Paris, Elsevier Masson, pp. 49-56.

Becker Gerhild, Xander Carola J., Blum Hubert E., Lutterbach Johannes, Momm Felix, Gysels Marjolein and Higginson Irene J. (2007) Do religious or spiritual beliefs influence bereavement? A systematic review, Palliative Medicine, 21 (3), pp. 207-217.

Berry John W. and Sam David L. (1997) Acculturation and adaptation, in John W. Berry, Marchall H. Segall and Çigdem Kagitçibasi Eds., Handbook of cross-cultural psychology (vol. 3), Boston, Allyn \& Bacon, pp. 291-326.

Bhugra Dinesh (2004) Migration and mental health, Acta Psychiatria Scandinavica, 109 (4), pp. 243-258.

Bhugra Dinesh and Gupta Susham (2011) Migration and Mental Health, New York, Cambridge University Press, 350 p.

Bolton Christopher and Camp Delpha J. (1987) Funeral rituals and the facilitation of grief work, Omega, 17 (4), pp. 343-352.

Bonanno George A. (2004) Loss, trauma and human resilience. Have we under-estimated our capacity to thrive after extremely aversive events? American Psychologist, 59 (1), pp. 20-28.

Bonanno George A. and Kaltman Stacey (2001) The varieties of grief experience, Clinical Psychology Review, 21 (5), pp. 705-734.

Bonanno George A., Neria Yuval, Mancini Anthony, Coifman Karin G., Litz Brett and Insel Beverly (2007) Is there more to complicated grief than depression and posttraumatic stress disorder? A test of incremental validity, Journal of Abnormal Psychology, 116 (2), pp. 342-351.

Boucebci Mahfoud (1994) Rang d'ainé dans la fratrie et risque psychopathologique : le syndrome d'aînité : Psychiatrie au Maghreb : destins d'enfants. Situations d'adolescence, L'Information psychiatrique, 70 (7), pp. 583-592. 
Carswell Kenneth, Blackburn Pennie and Barker Chris (2011) The Relationship between trauma, post-migration problems and the psychological well-being of refugees and asylum seekers, International Journal of Social Psychiatry, 57 (2), pp. 107-119.

Carta Mauro Giovanni, Bernal Mariola, Hardoy Maria Carolina, Haro-Abad Josep Maria and the "Report on the Mental Health in Europe" working group (2005) Migration and mental health in Europe (the state of the mental health in Europe working group: appendix 1), Clinical Practice and Epidemiology in Mental Health, 13 (1), pp. 1-16.

Casado Banghwa Lee, Hong Michin, Harrington Donna (2010) Measuring migratory grief and loss associated with the experience of immigration, Research on Social Work Practice, 20 (6), pp. 611-620.

Casado Banghwa Lee and Leung Patrick (2001) Migratory grief and depression among Elderly Chinese American immigrants, Journal of Gerontological Social Work, $36(1 / 2)$, pp. 5-26.

Chaïb Yassine (2006) Le rapatriement de la dépouille mortelle chez les immigrés maghrébins en France, L'autre, 7 (3), pp. 399-411.

Clément J.-P., Duveau F., Preux P.-M. et Léger J.-M. (2001) Mini-GDS chez les patients âgés suivis en médecine générale, L'Encéphale, 27, pp. 329-337.

Collins Wanda L. and Doolittle Amy (2006) Personal reflections of funeral rituals and spirituality in a Kentucky African American family, Death Studies, 30 (10), pp. 957-969.

Commission Vérité, Justice et Réconciliation (2012) Rapport final. Activités, Rapport d'investigations et recommandations (Vol 1), Lomé, Togo.

Craig Carlton D., Sossou Marie-Antoinette, Schnak Michele and Essex Heather (2008) Complicated grief and its relationship to mental health and well-being among Bosnian refugees after resettlement in the United States: Implications for practice, policy and research, Traumatology, 14 (4), pp. 103-115.

Creswell John W. and Plano Clark Vicki L. (2007) Designing and Conducting Mixed Methods Research, Thousand Oaks, CA, Sage, 275 p.

Crollard Allison, de Castro A.-B. and Tsai Jenny Hsin-Chun (2012) Occupational trajectories and immigrant worker health. Workplace, Health \& Safety, 60 (11), pp. 497-502.

Dassa Kolou S., Soedje Kokou M., Kokou-Kpolou Cyrille Kossigan et Gaba Dovi Adama G. (2012) Veuvage traditionnel et travail de deuil chez les Ewé du Togo, Annales africaines de Psychiatrie, 1 (1-2), pp. 87-97.

De Rosny Éric (2013) La résistance des rites traditionnels dans l'Afrique moderne, Théologiques, 4 (1), pp. 57-73.

Eisenbruch Maurice (1984a) Cross-cultural aspects of bereavement I: a conceptual framework for comparative analysis, Culture, Medicine and Psychiatry, 8 (4), pp. 283-309.

Eisenbruch Maurice (1984b) Cross-cultural aspects of bereavement. II: ethnic and cultural variations in the development of bereavement practices, Culture, Medicine and Psychiatry, 8 (4), pp. 315-347. 
Fenta Haile, Hyman Ilene, Rourke B. Sean, Moon Miea and Noh Samuel (2010) Somatic symptoms in a community sample of Ethiopian immigrants in Toronto, Canada, International Journal of Culture and Mental Health, 3 (1), pp. 1-15.

Fujisawa Daisuke, Miyashita Mitsunori, Nakajima Satomi, Ito Masaya, Kato Motoichiro and Kim Yoshiharu (2010) Prevalence and determinants of complicated grief in general population, Journal of Affective Disorders, 127, pp. 352-358.

Gana Karmel, Lesquoy Aurélie, Pariset Claire, de Tychey Claude et Bacqué MarieFrédérique (2004) L'échelle de Prigerson et al. : un dispositif d'évaluation préventive des complications du deuil?, in Claude de Tychey Éd., La prévention des dépressions, Paris, L'Harmattan, pp. 290-309.

Gayibor Nicoué L. (2011) Histoire des Togolais. Des origines aux années 1960. Tome 1. De I'histoire des origines à l'histoire du peuplement, Paris, Kartala, $716 \mathrm{p}$.

Gerritsen Annette A., Bramsen Inge, Devillé Walter, van Willigen Loes H., Hovens Johannes E. and van der Ploeg Henk M. (2006) Physical and mental health of Afghan, Iranian and Somali asylum seekers and refugees living in the Netherlands, Social Psychiatry and Psychiatric Epidemiology, 41, pp. 18-26.

Gierk Benjamin, Kohlmann Sebastian, Kroenke Kurt, Spangenberg Lena, Zenger Markus, Brähler Elmar and Löwe Bernd (2014) The Somatic Symptom Scale-8 (SSS-8) A Brief Measure of Somatic Symptom Burden, JAMA International Medecine, 174 (3), pp. 399-407.

Grinberg Leon and Grinberg Rebeca (1989) Psychoanalytic perspectives on migration and exile, New Haven, CT, Yale University Press, 240 p.

Hinton Devon E., Nickerson Aangela and Bryant Richard A. (2013a) Prolonged Grief in Cambodian Refugees Following Genocide: Rebirth Concerns and Avoidance of Reminders, Journal of Loss and Trauma, 18, pp. 444-460.

Hinton Devon E., Peou Sonith, Joshi Siddharth, Nickerson Aangela and Simon Naomi (2013b) Normal grief and complicated bereavement among traumatized Cambodian refugees: Cultural context and the central role of dreams of the dead, Culture, Medicine and Psychiatry, 37, pp. 427-464.

Holmes Thomas H. and Rahe Richard H. (1967). The social readjustment scale, Journal of Psychosomatic Research, 11 (2), pp. 213-218.

Karfo Kapouné, Thiam Mamadou H., Dassa Simliwa Kolou, Ouango Jean G. et Ouedraogo Arouna (2007) Aspects psychopathologiques de la dépression du sujet âgé en milieu africain au Burkina Faso, Perspectives Psy, 46 (2), pp. 159-164.

K'Delant Pascaline (2010) Le trouble du deuil persistant chez la personne âgée : évaluation et étude des effets de la personnalité, Université de Nancy, Thèse de doctorat en psychologie, document inédit, 264 p., [en ligne] consulté le 13/06/2011. URL : http://docnum.univ-lorraine.fr/public/-NANCY2/doc497/2010NAN21008_1. pdf

Kokou-Kpolou Cyrille Kossigan, Moukouta Charlemagne Simplice et Mbassa Menick Daniel (2016) Enjeux de la prise en charge du deuil pathologique en contexte migratoire chez le sujet togolais : aspects culturel et psychopathologique, in Charlemagne Simplice Moukouta Éd., Soins et migrations, Amiens, Licorne, pp. 171-195.

Kowalski Susan D. and Bondmass Mary D. (2008) Physiological and psychological symptoms of grief in widows, Research in Nursing \& Health, 31, pp. 23-30. 
Laban Cornelis J., Gernaat Hajo B., Komproe Ivan H., van der Tweel Ingeborg and De Jong Joop T. (2005) Postmigration living problems and commons psychiatric disorders in Iraqi asylum seekers in the Netherlands, Journal of Nervous Mental Disease, 193 (12), pp. 825-832.

Laurie Anna and Neimeyer Robert A. (2008) African Americans in bereavement: Grief as a function of ethnicity, Omega: Journal of Death and Dying, 57 (2), pp. 173-193.

Lee Ting-Yin (2010) The loss and grief in immigration: Pastoral Care for immigrants, Pastoral Psychology, 59 (2), pp. 159-169.

Levecque Katia and Van Rossem Ronan (2015) Depression in Europe: does migrant integration have mental health payoffs? A cross-national comparison of 20 European countries, Ethnicity \& Health, 20 (1), pp. 49-65.

Li Miao (2016) Pre-migration trauma and post-migration stressors for Asian and Latino American immigrants: Transnational stress proliferation, Social indicators Research, 129, pp. 47-59.

Lin Elizabeth, Carter William B. and Kleinman Arthur M. (1985) An exploration of somatization among Asian refugees and immigrants in primary care, American Journal of Public Health, 75, pp. 1080-1084.

Lobar Sandra L., Youngblut JoAnne M. and Brooten Dorothy (2006) Crosscultural beliefs, ceremonies, and rituals surrounding death of a loved one, Pediatric Nursing, 32 (1), pp. 44-50.

Lussier Martine (2011) Terre d'asile, terre de deuil : le travail psychique de l'exil, Paris, PUF, $256 \mathrm{p}$.

Lundorffa Marie, Holmgrena Helle, Zachariaeb Robert, Farver-Vestergaardb Ingeborg and O'Connora Maja (2017) Prevalence of prolonged grief disorder in adult bereavement: A systematic review and meta-analysis, Journal of Affective Disorders, 212, pp. 138-149.

Maciejewski Paul K., Maercker Andreas, Boelen Paul A. and Prigerson Holly G. (2016) "Prolonged grief disorder" and "persistent complex bereavement disorder", but not "complicated grief", are one and the same diagnostic entity: an analysis of data from the Yale Bereavement study, World Psychiatry, 15 (3), pp. 266-275.

Marie Alain (1997) Du sujet communautaire au sujet individuel, in Alain Marie Éd., L'Afrique des individus, Paris, Karthala, pp. 53-110.

Mazzucato Valentina, Kabki Mirjam and Smith Lothar (2006) Transnational Migration and the Economy of Funerals: Changing practices in Ghana, Development and Change, 37 (5), pp. 1047-1072.

Missinne Sarah and Bracke Piet (2012) Depressive symptoms among immigrants and ethnic minorities: a population based study in 23 European countries, Social psychiatry and psychiatric epidemiology, 47 (1), pp. 97-109.

Moukouta Charlemagne S, Mbassa Menick Daniel, Masson Joanic et Bernoussi Amal (2016) La clinique à l'épreuve des enjeux migratoires et culturels, L'Information psychiatrique, 92, pp. 1-7.

Nathan Tobie (1986) La folie des autres. Traité d'ethnopsychiatrie clinique, Paris, Dunod, $241 \mathrm{p}$. 
Newson Rachel S., Boelen Paul A., Hek Karin, Hofman Albert and Tiemeier Henning (2011) The prevalence and characteristics of complicated grief in older adults, Journal of Affective Disorders, 132 (1-2), pp. 231-238.

Panagiotopoulosa Georgia, Walkera Ruth and Luszczb Mary (2013) A comparison of widowhood and well-being among older Greek and British-Australian migrant women, Journal of Aging Studies, 27 (4), pp. 519-528.

Petit Agathe (2005) Des funérailles de l'entre-deux. Rituels funéraires des migrants Manjak en France, Archives de sciences sociales des religions, 131-132, pp. 87-99.

Prigerson Holly G., Horowitz Mardi J., Jacobs Selby C., Parkes Colin M., Aslan Mihaela et al. (2009) Prolonged grief disorder: psychometric validation criteria proposed for DSM-V and ICD 11, PloS Medicine, 6 (8), pp. 1-12.

Prigerson Holly G. and Jacobs Selby C. (2001) Traumatic grief as a distinct disorder: A rationale, consensus criteria, and a preliminary empirical test, in Margaret S. Stroebe, Robert O. Hansson, Henk Schut and Wolfgang Stroebe Eds., Handbook of bereavement research: Consequences, coping, and care, Washington, DC: American Psychological Association, pp. 613-645.

Prigerson Holly G., Maciejewski, Paul K., Reynolds III Charles F., Bierhals Andrew J., Newsom Jason T., Fasiczka Amy, Frank Ellen, Doman Jack and Miller Mark (1995) Inventory of Complicated Grief: A scale to measure maladaptive symptoms of loss, Psychiatry Research, 59 (1-2), pp. 65-79.

Pumariega Andrés J., Rothe Eugenio and Pumariega JoAnne B. (2005) Mental health of immigrants and refugees, Community Mental Health Journal, 41 (5), pp. 581-597.

Rachédi Lilyane, Le Gall Josiane et Leduc Véronique (2010) Réseaux transnationaux, familles immigrantes et deuils : réflexions pour la pratique, Lien social et Politiques, 64, pp. 175-187.

Rachédi Lilyane, Montgomery Catherine et Béatrice Halsouet (2016) Mort et deuil en contexte migratoire : spécificités, réseaux et entraide, Enfances, Familles, Générations, 24 [en ligne] consulté le 19/04/2017. URL : http://id.erudit. org/iderudit/1038114ar

Rachédi Lilyane et Tamouro Soumya (2012) Immigration et deuils : des réalités complexes, une nécessaire adaptation des services et des interventions, $L a$ Gérontoise, 23 (1), pp. 17-24.

Rando Therese (1993) The increasing prevalence of complicated mourning: the onslaught is just beginning, Omega, 26 (1), pp. 43-59.

Ritsner Michael, Ponizovsky Alexander, Kurs Rena and Modai Ilan (2000) Somatization in an immigrant population in Israel: a community survey of prevalence, risk factors, and help-seeking behavior, American Journal of Psychiatry, 157 (3), pp. 385-392.

Robben Antonius C. (2004) Death, Mourning and Burial: A Cross-cultural Reader, Malden, MA, Wiley-Blackwell, $336 \mathrm{p}$.

Robjant Katy, Hassan Rita and Katona Cornelius (2009) Mental health implications of detaining asylum seekers: systematic review, British Journal of Psychiatry, 194, pp. 306-312. 
Rohlof Hans G., Knipscheer Jeroen W. and Kleber Rolf J. (2014) Somatization in refugees: a review, Social Psychiatry and Psychiatric Epidemiology, 49 (11), pp 1793-1804.

Romanoff Bronna D. and Terenzio Marion (1998) Rituals and the grieving process, Death Studies, 22 (8), pp. 697-711.

Rosenblatt Paul C. (2008) Grief across cultures: A review and research agenda, in Margaret S. Stroebe, Robert O. Hansson, Henk Schut and Wolfgang Stroebe Eds., Handbook of bereavement research and practice: Advances in theory and intervention, Washington, DC, American Psychological Association, pp. 207-222.

Rosenblatt Paul C. (1993) Cross-cultural variation in the experience, expression, and understanding of grief, in Donald P. Irish, Kathleen F. Lundquist and Vivian J. Nelsen Eds., Ethnic variation in dying, death, and grief: Diversity in universality, Washington, DC, Taylor \& Francis, pp. 13-19.

Sanz Nikola (2012) La voix du corps. Essai sur les liens entre migration, deuil et somatisation, Université de Lausanne, Mémoire pour I'obtention du Master en Psychosociologie clinique, Document non publié, 137 p., [en ligne] consulté le 25/02/2017. URL : https://serval.unil.ch/resource/serval:BIB_S_000000017405. P001/REF

Sarkin Jeremy J. and Davi Tetevi (2017) The Togolese Truth, Justice and Reconciliation Commission: Lessons forTransitional Justice Processes Elsewhere, Peace and Conflict Studies, 24 (1), [en ligne] consulté le 20/04/2017. URL : http:// nsuworks.nova.edu/pcs/vol24/iss $1 / 2$

Segalen Martine (1998) Rites et rituels contemporains, Paris, Nathan, 128 p.

Thomas Louis-Vincent (1985) Rites de mort. Pour la paix des vivants, Paris, Fayard, $294 \mathrm{p}$.

Thomas Louis-Vincent (1975) Anthropologie de la mort, Paris, Payot, 538 p.

Tousignant Michel (1992) La santé mentale des migrants : analyse de son contexte social et longitudinal, Santé mentale au Québec, 17 (2), pp. 35-46.

Zisook Sidney, Reynolds III Charles F., Pies Ronald, Simon Naomi, Lebowitz Barry, Madowitz Jen, Tal-Young llanit and Shear Katherine (2010) Bereavement, complicated grief and DSM Part 1: Depression, The Journal of Clinical Psychiatry, 71 (7), pp. 955-956. 


\section{Cyrille Kossigan Kokou-Kpolou, Daniel Mbassa Menick, Charlemagne Simplice Moukouta et Élodie Gaëlle Ngameni}

\section{Étude du lien entre deuil, dépression et troubles somatiques auprès d'une population d'immigrés ouest-africains en Europe}

L'impact du deuil d'un être cher comme un des facteurs de risque majeurs associés aux troubles psychopathologiques et somatiques est jusqu'alors peu exploré. Cette étude a pour objectif d'examiner les facteurs de risque psychosociaux et culturels associés aux complications des réactions de deuil et aux symptômes dépressifs et somatiques chez les migrants et les réfugiés ouestafricains en Europe. L'étude a utilisé une méthodologie mixte et a inclus des participants qui ont perdu un parent ou proche parent. Les résultats ont montré que les immigrés qui sont des ainés de fratrie, des réfugiés et sans occupation professionnelle sont plus vulnérables aux complications du deuil, aux risques de dépression et aux symptômes somatiques. Les complications du deuil ainsi que le sentiment de culpabilité expliquent la somatisation, caractérisée par des douleurs articulaires, les douleurs thoraciques et des troubles de sommeil. Les immigrés qui ont participé aux rituels de deuil avaient manifesté moins de sentiment de culpabilité.

\section{Association between Mourning, Depression and Somatic Symptoms. Results from a Sample of West African Immigrants in Europe}

The impact of grief of parent as a risk factor associated with psychopathological and somatic disorders is hitherto unexplored. This study sought to examine the psychosocial and cultural risk factors associated with the complicated grief reactions, depressive and somatic symptoms among West African immigrants in Europe. The study combined qualitative and quantitative methods and included participants who lost a parent or a close relative. The finding showed that older siblings, refugees, unemployed immigrants were more vulnerable to complicated grief reactions, the risk of depression and somatic symptoms. The complicated grief reactions and feeling of guilt explained somatization, characterized by joint pain, chest pain, and sleep disorders. Immigrants who participated in the mourning rituals reportedly manifested less guilt.

\section{Estudio de la relación entre el duelo, la depresión y los trastornos somáticos entre una población de inmigrantes de África Occidental en Europa}

El impacto del duelo de un ser querido como uno de los principales factores de riesgo asociados con los trastornos psicopatológicos y somáticos ha sido poco explorado hasta ahora. El objetivo de este estudio fue examinar los factores de riesgo psicosociales y culturales asociados con las complicaciones de las reacciones de duelo y los síntomas depresivos y somáticos entre los migrantes y refugiados de África Occidental en Europa. El estudio utilizó una metodología mixta e incluyó participantes que habían perdido a uno de sus padres o parientes cercanos. Los resultados mostraron que los inmigrantes que eran hermanos mayores, refugiados y sin empleo eran más vulnerables a las complicaciones del duelo, los riesgos de depresión y los síntomas somáticos. Las complicaciones del duelo, así como el sentimiento de culpa explicaban la somatización, caracterizada por dolor en las articulaciones, dolor en el pecho y trastornos del sueño. Los inmigrantes que habían participado en los rituales de duelo habían mostrado menos sentimiento de culpabilidad. 